remarkable as to make this particular case equivalent, in value, to three cases. It is impossible to review the history of these cases without being convinced that the administration of ozonized oils has a most remarkable tendency to reduce the frequency of the pulse, in addition to producing a decided improvement in the general health and condition of the consumptive patient. In fourteen of the cases, sent by Dr. Thompson, there are only two in which no effect of this kind was produced. In the majority of instances the improvement was marked, and must be attributed only to the ozone, and not to the oil, since it was manifested in patients who had taken cod-liver oil and other oils, without any reduction in the frequency of the pulse; in some, under the use of simple oils the pulse was even accelerated, and under the administration of ozonized oil became immediately reduced. The effect on the pulse was nearly as distinct, when ozone was associated with cocoa-nut oil, or sun-flower oil, as it was with the cod-liver oil. The oils first mentioned produce no effect unless associated with ozone.

The reduction in the frequency of the pulse, produced by the ad. ministration of ozonized oils, is indeed remarkable. It is usually observable in two or three days. Dr. Thompson states, in connection with this subject, that "a reduction of twenty beats was observed, in certain cases, in two, three, four and six days : in other instances, there was a reduction of twenty-four pulsations in fourteen days; of thirty-four pulsations in thirteen days; of thirty-six pulsations in twenty-two days; and of forty pulsations in eleven days." In one patient, the pulse fell to 60 ; in most of the favorable cases, the reduction ceased when the normal standard was at. tained. This abstract, of Dr. Thompson, is of course taken from a large aggregate of cases. Such facts require no comment. We shall give none; for they demonstrate truths which cannot be misinterpreted or misunderstood.

[To be centinued.]

\title{
ON THE NORMAL MOTIONS OF THE HUMAN EYE.
}

By Prof. Hermaxy Helmholtz, For. Memb. R.S.

THE motions of the human eye are of considerable interest, as well for the physiology of voluntary muscular motion in general, as for the physiology of vision. Therefore I may be allowed to bring before this Society the results of some investigations relating to them, which I have made myself; and I may venture, perhaps, to hope that they are such as to interest not only physiologists and medical men, but every scientific man who desires to understand the mechanism of the perceptions of our senses.

The eyeball may be considered as a sphere, which can be turned round its centre as a fixed point. Although this description is not 
ahsolutely accurate, it is sufficiently so for our present purpose. The eyeball, indeed, is not fixed during its motion by the solid walls of an articular excavation, like the bone of the thigh; but, although it is surrounded at its posterior surface only by soft cellular tissue and fat, it cannot be moved in a perceptible degree forward and backward, because the volume of the cellular tissue, included between the eyeball and the osseous walls of the orbit, cannot be diminished or augmented by forces so feeble as the muscles of the eye are able to exert.

In the interior of the orbit, around the eyeball six muscles are situated, which can be employed to turn the eye round its centre. Four of them, the so-called recti muscles, are fastened at the hindmost point of the orbit, and go forward to fix themselves to the front part of the eyeball, passing over its widest circumference-or its equator, as we may call it, if we consider the foremost and hindmost points of the eyeball as its poles. These four recti muscles are from their position severally named superior, inferior, internal and external. Besides these, there are two oblique muscles, the ends of which come from the anterior margin of the orbit on the side next the nose, and, passing outwards, are attached at that side of the eyeball which is towards the temple-one of them, the superior oblique muscle, being stretched over the upper side of the eycball, the other, or inferior, going along its under side.

These six muscles can be combined as three pairs of antagonists. The internal and external recti turn the eye round a perpendicular axis, so that its visual line is directed either to the right side or to the left. The superior and inferior recti turn it round a horizontal axis, directed from the upper end of the nose to the temple; so that the superior rectus elevates the visual line, the inferior depresses it. Lastly, the oblique muscles turn the eye round an axis which is directed from its centre to the occiput, so that the superior oblique muscle lowers the visual line, and the inferior raises it; but these last two muscles not only raise and lower the visual line; they produce also a rotation of the eye round the visual line itself, of which we shall have to speak more afterwards.

A solid body, the centre of which is fixed, and which can be turned round three different axes of rotation, can be brought into every possible position consistent with the immobility of its centre. Look, for instance, at the motions of our arm, which are provided for at the shoulder-joint by the gliding of the very accurately spherical upper extremity of the humerus in the corresponding excavation of the scapula. When we stretch out the arm horizontally, we can turn it, first, round a perpendicular axis, moving it forwards and backwards; we can turn it, secondly, round a horizontal axis, raising it and lowering it; and, lastly, after having brought it by such motions into any direction we like, we can turn it round its own longitudinal axis, which goes from the shoulder to the hand; so that eren when the 
place of the hand in space is fixed, there are still certain different positions in which the arm can be turned.

Now let us see how far the motions of the eye can be compared to those of our arm. We can raise and lower the visual line, we can turn it to the left and to the right, we can bring it into every possible direction, throughout a certain range-as far, at least, as the connections of the eyeball permit. So far the motions of the eye are as free as those of the arm. But when we have chosen any determinate direction of the eye, can we turn the eye round the visual line as an axis, as we can turn the arm round its longitudinal axis?.

This is a question the answer to which is connected with a curions peculiarity of our voluntary motions. In a purely mechanical sense, we must answer this question in the affirmative. Yes, there exist muscles by the action of which those rotations round the visual line can be performed. But when we ask, "Can we do it by an act of our will?" we must answer, "No." We can voluntarily turn the visual line into every possible direction, but we cannot voluntarily use the muscles of our eye in such a way as to turn it round the visual line. Whenever the direction of the visual line is fixed, the position of our eye, as far as it depends upon our will, is completely fixed and cannot be altered.

This law was first satisfactorily proved by Professor Donders, of Utrecht, who, in a very ingenious way, controlled the position of the eye by those ocular spectra which remain in the field of vision after the eye had been fixed steadily during some time upon any brightly colored object. I have used for this purpose a diagram, of which the ground is grey paper, and in the middle is placed a narrow strip of red paper on a broader strip of green paper. The centre of the red strip is marked by two black points. When you look for about a minute steadily, and without moving your eye, at the centre of the diagram, the image of the colored strips is projected on the nervous membrane of your eye; those parts of this membrane on which the light falls are irritated, and in consequence of this irritation, their irritability is exhausted, they are fatigued, and they become less sensitive to that kind of light by which they were excited before. When you cease, therefore, to look at the colored strips, and turn your eye either to the grey ground of the diagram, or to any other part of the field of vision which is of a uniform feeble degree of illumination, you will see a spectrum of the colored strips, exhibiting the same apparent magnitude, but with colors reversed, a narrow green strip being in the middle of a broader red one. The cause of this appearance is, that those parts of your retina which were excited formerly by green light are less affected by the green rays contained in white or whitish light than by rays of the complementary color, and white light, therefore, appears to them reddish; to those parts of the nervous membrane, on the other hand, which had been fatigued by 
red light, white light afterwards appears to be greenish. The nervous membrane of the eye in these cases behaves nearly like the sensitive stratum in a photographic apparatus, which is altered by light during the exposure in such a way that it is impressed differently afterwards by various agents; and the impression of light on the retina may be, perhaps, of the same essential nature as the im. pression made upon a photographic plate. But the impressiom made on the living eye does not last so long as that on sensitive compounds of silver; it vanishes very soon if the light be not too strong. Light of great intensity, like that of the sun when directly looked at, can develope very dark ocular spectra, which last a quarter of an hour, or even longer, and disturb the perception of external objects very much, as is well known. One must be very careful to avoid the use of too strong a light in these experiments, because the nervous apparatus of the eye is easily injured by it; and the brightness of these colored strips, when illuminated by common daylight, is quite sufficient for our present purpose.

Now you will perceive easily that these ocular spectra are extremely well adapted to ascertain the position of the eyeball, because they have a fixed connection with certain parts of the retina itself. If the eyeball could turn about its visual line as an axis, the ocular spectrum would apparently undergo the same degree of rotation; and hence, when we move about the eye, and at last return to the same direction of the visual line, we can recognize easily and accurately whether the eye has returned into the same position as before, or whether the degree of its rotation round the visual line has been altered. Professor Donders has proved, by using this very delicate test, that the human eye, in its normal state, returns always into the same position when the visual line is brought into the same direction. The position and direction of the eye are to be determined in this case in reference to the head of the observer; and I beg you to understand always, when I say that the eye or its visual line is moved upwards or downwards, that it is moved either in the direction of the forehead or in that of the cheek; and when I say it is moved to the left or to the right, you are to understand the left or right side of the head. Therefore, when the head itself is not in its common vertical position, the rertical line here understood is not accordant with the line of the plummet.

Before the researches of Donders, some obserrers believed they had found a difference in the relative positions of the ese, when the head was brought into different situations. They had used either small brown spots of the iris, or red vessels in the white of the eye, to ascertain the real position of the eyeball; but their apparent results have been shown to be erroneous by the much more trustworthy method of Donders.

In the first place, therefore, we may state that the position of the cyeball depends exclusively upon the direction of the visual line in 
reference to the position of the head of the observer. But now we must ask, what is the law regulating the position of the eye for every direction of its visual line? In order to define this law, we must first notice that there exists a certain direction of the visual line, which, in relation to the motions of the eye, is distinguished from all other directions of the eye; and we may call it the central or primary direction of the visual line. This direction is parallel to the median vertical plane of the head; and it is horizontal when the head of the observer, who is standing, is kept in a convenient erect position to look at distant points of the horizon. How this primary direction of the visual line may be determined practically with greater accuracy we shall see afterwards. All other directions of the visual line we may call sccondary directions.

A plane which passes through the visual line of the eye, I call $a$ meridian plane of the eyc. Such a plane cuts through the retina in a certain line; and when the eye has been moved, we consider as the same meridian plane that plane which passes through the new direction of the visual line and the same points of the retina as before.

After having given these definitions, we may express the law of the motions of the eye in the following way:-

Whencrer the eyc is brought into a secondary position, that meridian plane of the eye which goes through the primary direction of the visual line has the same position as it has in the primary direction of the eye.

It follows from this law that the secondary position of the eye may be found also by turning the eye from its primary position round a fixed axis which is normal as well to the primary as to the secondary of the visual line.

It would take too long to explain the different ways in which different observers have tried to determine the law of the motions of the eyeball. They have employed complicated apparatus for determining the angles by which the direction and the rotation of the eye were to be measured. But usually two difficulties arise from the use of such instruments containing graduated circles, in the centre of which the eye must be kept steady. In the first place, it is very difficult to fix the head of the observer so firmly that he cannot alter its position during a continuous series of observations, and that he reassumes exactly the same position of the head when he returns to his measurements after a pause-conditions which must necessarily be fulfilled if the observations are to agree with each other. Secondly, I have found that the eye must not be kept too long a time in a direction which is near to the limits of the field of vision; else its muscles are fatigued, and the positions of the eyeball corresponding to different directions of the visual line are somewhat altered. But if we have to measure angles on graduated circles, it is difficult to avoid keeping the eye too long in directions deviating far from the primary direction. 
I think that it depended upon these causes, that the observations carried out by Meissner, Fick and Wundt agreed very ill with each other and with the law which I have explained above, and which was first stated by Professor Listing of Göttingen, but without any experimental proof. Happily, it is possible, as I found out, to prove the validity of this law by a very simple method, which is not subject to those sources of error I have named, and which I may be allowed to explain briefly.

In order to steady the attitude of the head in reference to the direction of the visual line, I have taken a little wooden board, one end of which is hollowed into a curve fitting the arch of the human teeth; the margin of this hollow is covered with scaling wax, into which, after it had been softened by heat and had been cooled again sufficiently, I inserted both series of my teeth, so that I kept it firmly between my jaws. The impressions of the teeth remain indented in the sealing-wax; and when I put my teeth afterwards into these impressions, I am sure that the little board is brought exactly into the same position, relatively to my head and my eyes, as it was before. On the other end of that little board, which is kept horizontally between the teeth, a vertical piece of wood is fastened, on which I fix horizontally a little strip of card pointed at each end, so that these two points are situated about five inches before my eyes, one before the right eye, the other before the left. The length of the strip of card must be equal to the distance between the centres of the eyes, which is 68 millimetres for my own eyes. Looking now with the right eye in the direction of the right point of that strip, and with the left eye in the direction of the left point, I am sure to bring the eyes always into the same position relatively to my head, so long as the position of the strip of card on the wooden picce remains unaltered.

As a field of vision I use either a wall covered with a grey paper, in the pattern of which horizontal and rertical lines can be easily perceived, or a drawing-board covered with grey drawing-paper, on which a system of horizontal and vertical lines is drawn, and color. ed stripes are fastened along a central perpendicular line $a b$.

Now the observer at first must endeavor to find out that position of his eyes which we call the primary position. In order to do this, the observer takes the wooden piece between his teeth, and brings his head into such a position that his right eye looks to the centre of the colored stripes, in a direction perpendicular to the plane of the drawing. Then he brings his head into such an attitude that the right end of the card-strip appears in the same direction as the centre of the colored stripe. After having steadily looked for some time to the middle of the colored stripe, he turns away his gaze to the end of either the vertical or horizontal lines, which are drawn through the centre of the colored stripe. There he will see an ocular spectrum of the colored stripe, and will observe if it coincides VoL. LXXI.-No. 14A 
with the horizontal lines of the drawing. If not, he must alter the position of the strip of card on the wooden bar to which it is fastened, till he finds that the ocular spectrum of the colored stripe remains horizontal when any point either of the line $a b$ or the central horizontal line $c d$ is looked at. When he has thus found the primary direction of his visual line for the right eye, he docs the same for the left.

The ocular spectra soon ranish, but they are easily renewed by looking again to the centre of the stripes. Care must be taken that the observer looks always in a direction perpendicular to the plane of the drawing whenever he looks to the centre of the colored stripe, and that he does not move his head. If he should have moved it, he would find it out immediately when he looks back to the strip, because the point of the card-strip would no longer corer the centre of the colored stripe.

So you see that the primary direction of the visual line is completely fixed, and that the eye, which wants only to glance for an instant at a peripheral point of the drawing, and then goes back again to the centre, is not fatigued.

This method of finding the primary position of the eye proves at the same time that vertical and horizontal lines keep their vertical or horizontal position in the field of vision when the eye is moved from its primary direction rertically or horizontally; and you see, therefore, that these movements agree with the law which I have enunciated. That is to say, during vertical movements of the eye the vertical meridian plane keeps its vertical position. and during horizontal movements the horizontal meridian.

Now you need only bring either your own head into an inclined position, or the diagram with the lines, and repeat the experiment, putting your head at first into such a position that the centre of the diagram corresponds with the primary direction of the visual line, and moving afterwards the eye along the lines $a b$ or $c d$, and you will find the ocular spectrum of the colored line coinciding with those black lines which are parallel with $a . b$. In this way, therefore, you can easily prove the law of Listing for every possible direction of the visual line.

I found the results of these experiments in complete agreement with the law of Listing for my own eyes, and for those of several other persons with normal power of vision. The eyes of very shortsighted persons, on the contrary, often show irregularities, which unay be caused by the elongation of the posterior part of those eyes.

These motions of our eyes are a peculiar instance of motions, which, being quite voluntary, and produced by the action of our will, are nevcrtheless limited as regards their extent and their combinations. We find similar limitations of motion of the eyes in other cases also. We cannot turn one eye up, the other down; we cannot move both eyes at the same time to the outer angle; we are obliged 
to combine always a certain degree of accommodation of the eyes to distance, with a certain angle of convergence of their axes. In these latter cases it can be proved that the faculty of producing these motions is given to our will, although our will is not commonly capable of using this faculty. We have come by experience to move our eyes with great dexterity and readiness, so that we see any visible object at the same time single and as accurately as possible; this is the only end which we have learnt to reach by muscular exertion; but we have not learnt to bring our eyes into any given position. In order to move them to the right, we must look to an object situated on our right side, or imagine such an object and search for it with our eyes. We can move them both inwards, but only when we strive to look at the back of our nose, or at an imaginary object situated near that place. But commonly there is no object which could be seen single by turning one eye upwards, the other downwards, or both of them outwards, and we are therefore unable to bring our eyes into such positions. But it is a well-known fact, that when we look at stercoscopic pictures, and increase the distance of the pictures by degrees, our eycs follow the motion of the pictures, and that we are able to combine them into an apparently single object, although our eyes are obliged to turn into diverging directions. Professor Donders, as well as myself, has found that when we look to a distant object, and put before one of our eyes a prism of glass, the refracting angle of which is between 3 and 6 degrees, and turn the prism at first into such a position before the eye that its angle looks to the nose and the visual lines converge, we are alle to turn the prism slowly, so that its angle looks upwards or downwards, keep. ing all this time the object apparently single at which we look. But when we take away the prism, so that the eyes must return to their normal position before they can sce the object single, we sce the object double for a short time-one image higher than the other. The images approach after some seconds of time and unite at last into one.

By these experiments it is proved that we can more both eyes outward, or one up and the other down, when we use them under such conditions that such a position is required in order that we may see the objects single at which we are looking.

I have sometimes remarked that I saw double images of single objects, when I was sleepy and tried to keep myself awake. Of these images one was sometimes higher than the other, and sometimes they were crossed, one of them being rotated round the visual line. In this state of the brain, therefore, where our will begins to lose its power, and our muscles are left to mere involuntary and mechanical impulses, an abnormal rotation of the eye round the visual line is possible. I infer also from this observation, that the rotation of the eye round the visual axis cannot be effected by our will, because we have not learnt by which exertion of our will we are to effect it, 
and that the inability does not depend on any anatomical structure either of our nerves or of our muscles which limits the combination of motion. We should expect, on the contrary, that, if such an anatomical mechanism existed, it should come out more distinctly when the will has lost its power.

We may ask, therefore, if this peculiar manner of moving the eyes, which is determined by the law of Listing, is produced by practical exercise on account of its affording any advantages to visual perceptions. And I beliere that certain advantages are indeed connected with it.

We cannot rotate our eses in the head, but we can rotate the head with the cyes. When we perform such a motion, looking steadily to the same point, we remark that the visible objects turn apparently a little round the fixed point, and we lose by such a motion of our eye the perception of the steadiness of the objects at which we look. Every position of the visual line is connected with a determined and constant degree of rotation, according to the law of Donders; and in altering this rotation we should judge the position of external objects wrongly.

The same will take place when we change the direction of the visual line. Suppose the amplitude of such motions to be infinitely small; then we may consider this part of the field of vision, and the corresponding part of the retina on which it is projected, as plane surfaces. If during any motion of the eye the optic image is displaced so that in its new position it remains parallel to its former position on the retina, we shall have no apparent motions of the objects. When, on the contrary, the optic image of the visible objects is dislocated so that it is not parallel to its former position on the retina, we must expect to perceive an apparent rotation of the objects.

As long as the motions of the eye describe infinitely small angles, the eye can be moved in such a way that the optic image remains always parallel to its first position. For this end the eye must be turned round axes of rotation which are perpendicular to the visual line; and we see indeed that this is done, according to the law of Listing, when the eye is moving near its primary position. But it is not possible to fulfil this condition completely when the eye is moved through a wider area which comprises a larger part of the spherical field of view. For if we were to turn the eye always round an axis perpendicular to the visual line, it would come into very different positions after having been turned through different ways to the same final direction.

The fault, therefore, which we should strive to avoid in the motions of our eye, cannot be completely avoided, but it can be made as small as possible for the whole field of vision.

The problem, to find such a law for the motions of the eye that the sum of all the rotations round the visual line for all possible infinitely 
small motions of the eye throughout the whole field of vision becomes a minimum, is a problem to be solved by the calculus of variations. I have found that the solution for a circular field of vision, which corresponds nearly to the forms of the actual field of vision, gives indeed the law of Listing.

I conclude from these researches, that the actual mode of moving the eye is that mode by which the perception of the steadiness of the objects through the whole field of vision can be kept up the best; and I suppose, therefore, that this mode of motion is produced by experience and exercise, because it is the best suited for accurate perception of the position of external objects.

But in this mode of moving, rotations round the visual line are not completely aroided when the eye is moved in a circular direction round the primary position of the visual line; and it is easy to recognize that in such a case we are subject to optical illusions.

Turn your eyes to a horizontal line situated in the highest part of the field of vision, and let them follow this line from one end to the other. The line will appear like a curved line, the convexity of which looks downward. When you look to its right extremity, it seems to rise from the left to the right; when you look to the left extremity of the line, the left end seems to rise. In the same way, all straight lines which go through the peripheral parts of the field of vision appear to be curved, and to change their position a little, if you look to their upper or their lower ends.-Proceedings of the Royal Society.

[To be continued.]

\section{THE BOSTON MEDICAL AND SURGICAL JOURNAL.}

BOSTON: THURSDAY, NOVEMBER 3, 1864.

Proceedings of the Nineteenth Annual Meeting of the Ohio State Medicat Society, June 21st and 22D, 1864.-A pamphlet of some 90 pages is rather a small annual contribution from the Society of so large a State as Ohio, but better a small publication, if it be a good one, than a large, dilute, watery one. The one before us contains several papers of merit and practical value.

The Valedictory Address by the retiring President, Dr. W. P. Kincaid, is in the usual strain of such compositions. It is to our minds a positively cruel custom which exists in some of our State Societies, which compels the retiring President, on each recurring anniversary, to reiterate the old commonplaces pertaining to the dignity, the great responsibilities and high moral standing of the medical profession, and to make an effort at eloquence in attempting to stir up his audience to a fresh appreciation of them. In the address before us, the author bestows the usual attention on these topics, and dwells with a good deal of emphasis, also, on the value of a general education to a phy- 